\title{
STRENGTHENING OF SYNAPTIC CONTACTS OF AN EXCITATORY AXON ON ELIMINATION OF A SECOND EXCITATORY AXON INNERVATING THE SAME TARGET ${ }^{1}$
}

\author{
I. PARNAS, ${ }^{*, 2}$ J. DUDEL, $\ddagger$ I. COHEN,* AND CH. FRANKE $\ddagger$ \\ * Department of Neurobiology, Hebrew University, Jerusalem, Israel and $\ddagger$ Physiologisches Institüt der Technischen Universität \\ München, Biedersteiner strasse 29, D-8000 München 40, Federal Republic of Germany
}

Received September 26, 1983; Revised February 6, 1984; Accepted February 7, 1984

\begin{abstract}
In the deep abdominal extensor muscles of spiny lobsters (Panulirus -pennicillatus), the common excitor axon of segment II was eliminated by intracellular injection of pronase. At 1 to 23 days after the operation, the quantal content of excitatory postsynaptic currents (EPSCs), elicited by stimulation of the specific excitor of the $L_{1}$ muscle, was determined in a specific area of the $L_{1}$ muscle, both in the operated and in the contralateral control side. The EPSCs in the operated muscles had about a 5 times higher quantal content compared to those in the controls, the change developing within 1 to 2 days after operation. In camera lucida drawings of preparations stained with methylene blue, increased branching of the remaining excitatory axon was obvious at more than 4 days after the operation.

To investigate the possibility of contribution of central mechanisms (Rotshenker, S. (1979) J. Physiol. (Lond.) 292: 535-547). to this effect, the bundle of five axons to the deep abdominal extensors of segment II was cut immediately after injection of pronase into the common excitor axon. This caused a reduction of the quantal content of EPSCs and shrinking of the field of innervation in the operated $\mathrm{L}_{1}$ muscle as compared to the control. Therefore, axonal continuity or central connections seem to be necessary for the development of an increased innervation by the specific excitor to $\mathrm{L}_{1}$ after eliminating the common excitor axon.

Possible postsynaptic effects of the elimination of the common excitor axon were controlled by recording synaptic single channel currents elicited by the excitatory transmitter glutamate, using the patch clamp method. These single current events did not show appreciable changes in operated $\mathrm{L}_{1}$ muscles. Therefore, the presynaptic strengthening effect on the nerve terminals of the specific $\mathrm{L}_{1}$ excitor is predominant after elimination of the common excitor axon.
\end{abstract}

The nature of trophic interactions between nerve cells and their target and the organizational influences between nerve cells innervating the same target have been investigated extensively (Purves, 1977). As a result, the concepts of competition between neurons innervating the same target (Purves and Lichtman, 1978, 1980; Hume and Purves, 1983), elimination of supernumerary inner-

\footnotetext{
${ }^{1}$ We wish to thank the Egyptian authorities, especially the Consul Mr. H. Issa, for cooperation in the collection of lobsters on their territory, and Dr. Avi Baranes, representing the staff of the Steinitz Marine Biological Laboratory. We further want to thank Mr. D. Beyer for mechanical, Mr. W. Zeitz for electronic, Mrs. I. Horstmann for technical, and Mrs. M. Griessl for secretarial help. The critical reading of the manuscript and useful comments of Professor R. Werman are greatly apprecited. This work was supported by facilities of the Steinitz Marine Laboratory of the Hebrew University in Eilat, Israel, and by a grant from the Deutsche Forschungsgemeinshaft.

${ }^{2}$ To whom correspondence should be addressed.
}

vation (Brown et al., 1976; Haimann et al., 1981), and the development of weak or strong synapses (Hume and Purves, 1983) have been proposed. Many of these studies were made on the vertebrate neuromuscular junction, in which only one type of afferent axon and one neurotransmitter are involved. In the central nervous system different types of axons approach one cell, and it would be of great interest to understand organizational interactions in such more complicated systems.

The neuromuscular system of Crustacea, innervated by few but functionally specialized axons of excitatory and inhibitory ("fast" and "slow") types (Atwood, 1977), offers an opportunity to study organizational effects at a higher level of complexity. However, it was found that peripheral stumps of cut axons survived for many months in the crayfish opener muscle (Hoy et al., 1967; Hoy, 1969). Later it was shown that degeneration of the axon to the accessory flexor muscle of a crayfish can be pro- 
duced, but no supersensitivity to the excitatory transmitter, glutamate, or other striking effects on nerve or muscle properties have been observed (Bittner and Johnson, 1974; Frank, 1974). Advances were possible with the technique of elimination of single axons by intracellular injection of pronase as developed for neurons of the leech (Parnas and Bowling, 1977; Bowling et al., 1978). When the common inhibitory axon to the deep abdominal extensor muscles of lobsters (Parnas and Atwood, 1966) was eliminted by pronase injection, the remaining excitatory axons elicited prolonged synaptic potentials which were at least partially the result of a prolonged open time of postsynaptic channels (Dudel et al., 1981; I. Parnas et al; 1982a; Ch. Franke, I. Cohen, J. Dudel, and I. Parnas, manuscript in preparation). This was the first demonstration of interactions between axons of different functional type and releasing different transmitters.

In the present paper our studies have been extended to the effects of elimination of the common excitatory axon in the same abdominal extensor muscle of lobster. Whereas the consequences of elimination of the inhibitory axon were mainly postsynaptic, removal of the common excitatory axon led to surprisingly strong presynaptic alterations.

\section{Materials and Methods}

The experiments were performed in the Steinitz Marine Biological Laboratory in Eilat, Israel, on the spiny lobster (Panulirus pennicillatus) collected in the Gulf of Eilat. The animals were kept in running sea water in tanks; freshly operated animals were held in separate tanks. The common excitatory axon of the deep abdominal extensor muscles was eliminated by pressure injection of pronase as described by I. Parnas et al. (1982a). The animals were anesthetized by packing them in sea water ice. A small window was cut into the dorsal carapace of abdominal segment II, and the nerve bundle containing the five axons to the muscle (Fig. 1) was exposed. The largest axon is the common excitor. The axon was penetrated with a microelectrode, and pronase was injected. After closing the carapace, the lobster was rewarmed slowly, which appeared to improve the rate of survival.

After a period of 1 to 23 days, the deep abdominal extensor muscles of segment II (and often also segment III) were isolated. The muscles on both sides of the animal were used, the ones on the nonoperated side serving as controls. The preparation consists of the medial muscle $(M)$ and the two lateral muscles $\left(L_{1}\right.$ and $\left.L_{2}\right)$ with their axons, as represented schematically in Figure 1. A more realistic drawing of the preparation was given by Parnas and Atwood (1966). The preparation was pinned down in a chamber with a Sylgard floor and lightguide bottom illumination and was superfused with solution at 14 to $15^{\circ} \mathrm{C}$. The normal solution contained: $\mathrm{NaCl}, 520 \mathrm{~mm} ; \mathrm{KCl}, 12 \mathrm{~mm} ; \mathrm{CaCl}_{2}, 12 \mathrm{mM} \mathrm{MgCl}_{2}, 10$ mM; Tris-chloride, $2.5 \mathrm{mM}$, pH 7.5. The axons could be stimulated selectively through a suction electrode with a tip about $50 \mu \mathrm{m}$ in diameter placed directly on the respective axon.

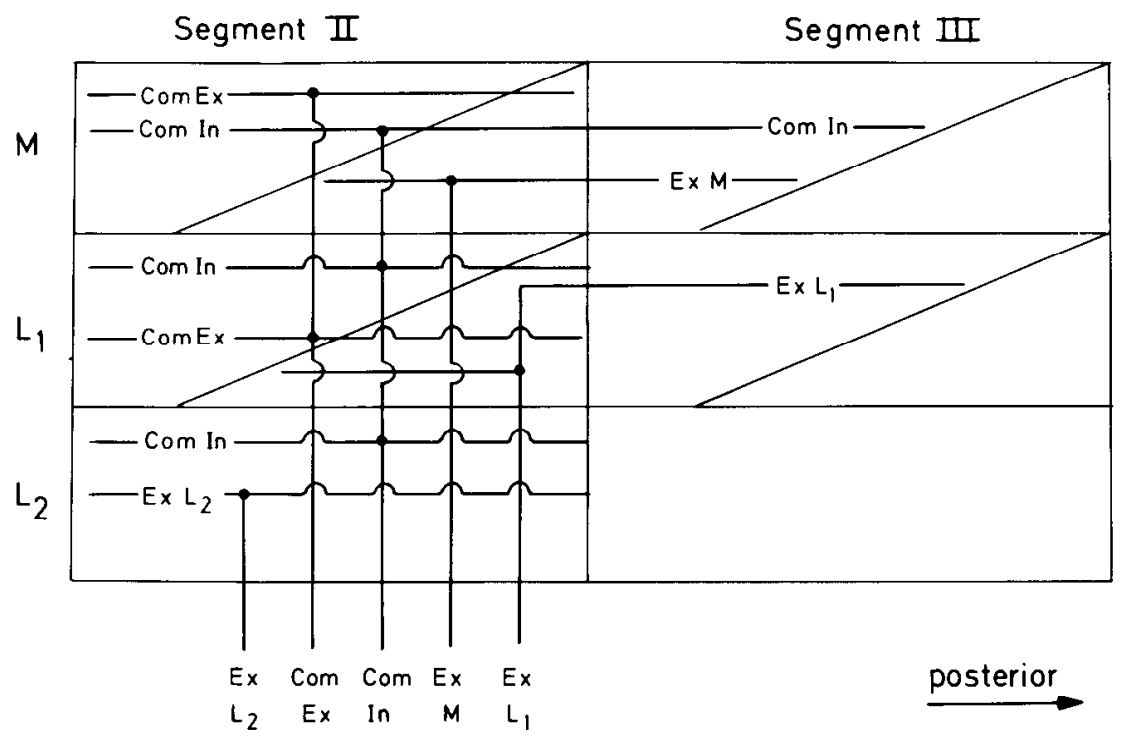

Figure 1. Scheme of distribution of the axons entering the deep abdominal extensor muscle at segment II. The muscles consist of the medial $(M)$ bundle and the two $\left(L_{1}\right.$ and $L_{2}$ ) lateral bundles. In both $M$ and $L_{1}$, the muscle fibers can be separated to an anterior part and a posterior part by the directions of the muscle fibers which are twisted, forming a clear division line which runs obliquely. In the following figures this line is marked as "partition." The axons are the specific excitors of $\mathrm{L}_{1}\left(E x L_{1}\right)$ and of $\mathrm{M}(E x M)$, the common inhibitor (Com In) and excitor (Com $E x)$, and the specific excitor of $\mathrm{L}_{2}\left(E x L_{2}\right) . E x L_{1}$ and $E x M$ of segment II innervate only the posterior portions of the respective muscles in segment II, but in addition also the anterior portions of the respective muscles in segment III. The common inhibitor in $M$ also crosses to the anterior portion of $M$ in segment III. A more realistic scheme is given by Parnas and Atwood (1966). 
In each experiment we initially checked whether the common excitatory axon had been injected successfully. This was necessary because errors of identification occurred when injecting pronase. In addition, a few injections were not successful. In the isolated and well illuminated preparation, the fact that one axon had been destroyed could be seen clearly with a dissecting microscope, and usually this axon could be identified visually due to its position relative to the remaining axons. However, the function of the remaining axons was always ascertained by selective stimulation and extracellular recording from the respective muscles (see below). The specific excitors of the medial and the two lateral muscles $\mathrm{L}_{1}$ and $\mathrm{L}_{2}$ were identified easily because synaptic currents were only seen in the respective muscle (Fig. 1). Stimulation of the common excitor elicited synaptic currents in the medial and the $\mathrm{L}_{1}$ muscles. The main focus of interest of the experiments was the $L_{1}$ muscle. In this muscle, the discrimination between the common excitor and the specific $L_{1}$ excitor was further aided by the anatomical distribution of the specific $\mathrm{L}_{1}$ excitor, which innervates only the posterior part of the $L_{1}$ muscle of segment II and in addition the anterior part of segment III (Fig. 1). The posterior and anterior parts of the $I_{\iota_{1}}$ muscle are separated by a line which runs obliquely across the muscle (Parnas and Atwood, 1966) (see also Fig. 5). Therefore, synaptic activity is observed all over the $\mathrm{L}_{1}$ muscle of segment II on stimulating the common excitor, but only in the posterior part on stimulating the $\mathrm{L}_{1}$ excitor. At this posterior region each muscle fiber is thus innervated by two excitatory axons from the same segment. Finally, stimulation of the common inhibitor led to low level synaptic activity on all muscles but not to contractions. Stimulation of the common excitor axon led to vigorous contractions. When it was ascertained that the common excitor axon had been eliminated and that the other axons were functioning, the stimulation electrode was placed on the $\mathrm{L}_{1}$ excitor axon during the further course of the experiment.

Quantal synaptic currents elicited by nerve stimulation were recorded with a macro-patch clamp technique modified from the patch clamp suggested by Neher et al. (1978). This technique has been used extensively on crayfish muscle (Dudel, 1981; H. Parnas et al., 1982; I. Parnas et al., 1982b). Optimal electrodes for the present preparation had smaller tips than those used in the crayfish studies: the fire-polished tips had 10 - to $30-\mu \mathrm{m}$ openings and 0.7 to 1.5 megohms of resistance. They were filled with bathing solution.

In some of the muscles, single excitatory channels were also recorded with the micro-patch clamp technique as developed by Neher et al. (1978) and adapted to crustacean muscle by Franke et al. (Ch. Franke, J. Dudel, and W. Finger, submitted for publication). In these recordings electrodes with 1 - to $2-\mu \mathrm{m}$ openings and 3 to 5 megohms of resistance, filled with bathing solution containing $0.2 \mathrm{M}$ glutamate, were employed. The muscles were treated with collagenase ( 1 to $2 \mathrm{mg} / \mathrm{ml}$ ) for about 1 to $1.5 \mathrm{hr}$ before recording and also with concanavalin $\mathrm{A}$ (Con A) in order to prevent desensitization of the receptors (Dudel, 1979; Mathers, 1981). The seal resistances were more than 100 megohms. The recordings were in- spected on line on a digital oscilloscope (Nicolet Explorer 1) and recorded on FM analogue tape (Hewlett Packard $3964 \mathrm{~A}$ ). They were evaluated later from the tape using a Nicolet Med 80 computer system.

\section{Results}

Excitatory synaptic currents (EPSCs) in normal muscles and in muscles in which the common excitor had been removed. EPSCs elicited by stimulation of the $\mathrm{L}_{1}$ excitor were recorded extracellularly at many sites on the $L_{1}$ muscle. The quality of the recordings is shown in Figure 2. Six single recordings are represented, together with an average of 128 EPSCs at the same site. 'Three of the single EPSCs are "failures," the second and the sixth represent single quanta, and the third contains two or three quanta. The exact placing of the electrode on a synaptic site is crucial; with slight movement of the electrode (a few micra), positive currents were seen indicating recording from a distant synapse (Dudel, 1981). The quantal content of EPSCs was evaluated directly while recording; the number of failures to release $\left(N_{0}\right)$

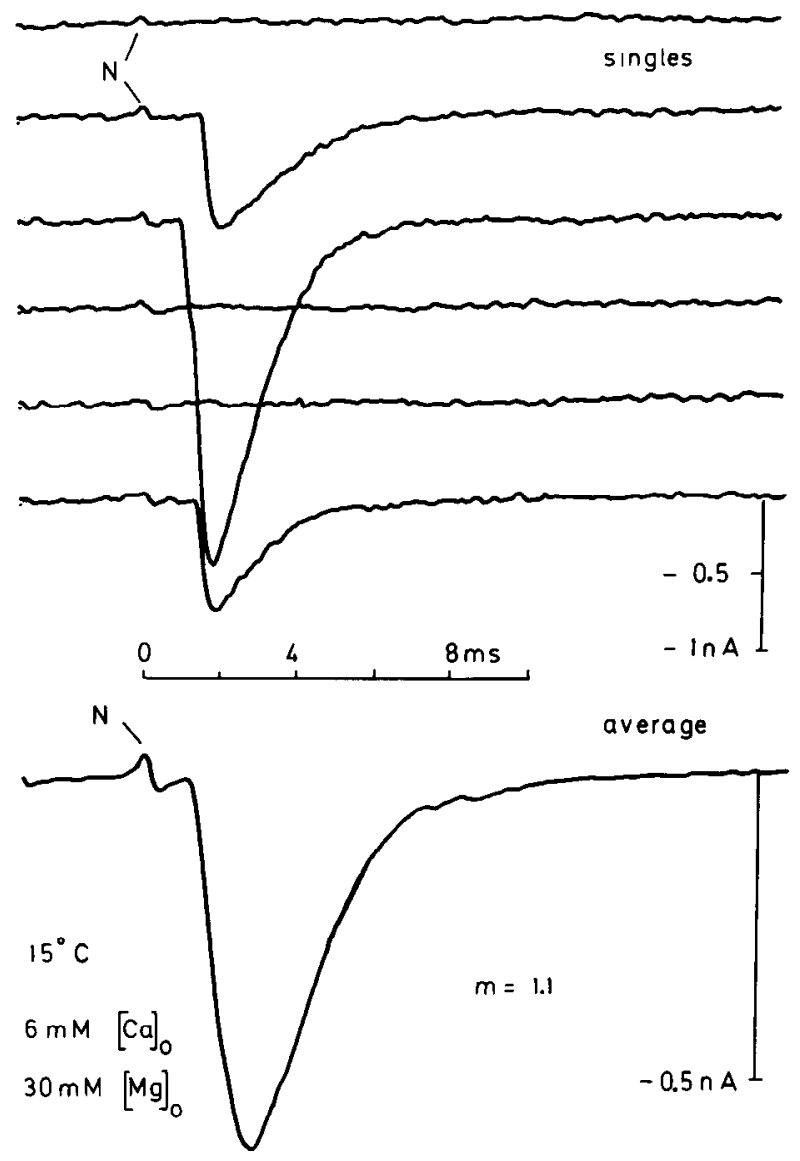

Figure 2. Measurement of EPSCs. The upper part shows six single traces. In each, a record from the nerve terminal $(N)$ is seen, but in three sweeps no release occurs. The second and the sixlh traces show a unitary response. The lower part shows the average of 128 such traces at the same synaptic site. The quantal content $(m)$ of this average EPSC was 1.1, as calculated from the proportion of the observed release and failures. Amplitude scales are different in singles and in average; time bases are the same. The EPSCs were recorded at $15^{\circ} \mathrm{C}$ and in low $[\mathrm{Ca}]_{0}=6 \mathrm{mM}$ and high $[\mathrm{Mg}]_{0}=30 \mathrm{mM}$. 
was counted and related to the total number of stimuli $(N)$, which was usually 128 . If Poison statistics are assumed (Del Castillo and Katz, 1954; Johnson and Wernig, 1971), the average quantal content is $m=\ln$ $\left(N / N_{0}\right)$. The EPSCs were averaged in parallel and, from the average and $m$, the size of the average single quantum could be obtained. Under experimental conditions like those in the experiment of Figure 2, the average quantum size was usually between -0.5 and $-1.2 \mathrm{nA}$. In normal bathing solution, the muscle often contracted strongly on stimulation, and the EPSCs had quantal contents, $m$ $>2$, which could only be evaluated with difficulty. Therefore, most experiments described below were performed at $6 \mathrm{~mm}[\mathrm{Ca}]_{0}$ and $30 \mathrm{mM}[\mathrm{Mg}]_{0}$. As is well known (Jenkinson, 1957; Katz and Miledi, 1965; Dudel et al., 1982; H. Parnas et al., 1982; I. Parnas et al., 1982b), lowering $[\mathrm{Ca}]_{0}$ and increasing $[\mathrm{Mg}]_{n}$ reduced the release rates, generally to below $m=1$ in normal $\mathrm{L}_{1}$ muscles.

Comparing excitatory synaptic transmission on stimulation of the $\mathrm{L}_{1}$ axon in normal muscles and in those in which the common excitor axon had been removed, it was obvious that transmission was improved in the operated muscles. In order to estimate this improvement quantitatively, an experimental routine was developed in which the EPSCs were recorded at 8 to 20 sites in analogous, well defined regions of the nonoperated and of the operated $L_{1}$ muscle. At each of these sites the quantal content of the EPSC was determined, using the same standard conditions and the same electrode. Figure 3 shows a schematic drawing of the fibers of the $L_{1}$ muscle in the region in which axons enter or cross the muscle. The investigation concentrated on the region between the main branch of the $L_{1}$ axon, which runs in the nerve bundle crossing $\mathrm{L}_{1}$ to the medial muscle (Fig. 3 , right hand branch on nonoperated side), and the posterior branch of $L_{1}$ excitor which innervates segment III (Fig. 1). The field of recordings was further limited to the posterior part of the $\mathrm{L}_{1}$ muscle (Fig. 1), which is separated from the anterior part by the oblique line indicated in the upper portions of the drawings of Figure 3. Recordings were restricted to these special regions in order to make results obtained from the nonoperated and the operated sides of the $\mathrm{L}_{1}$ comparable. However, these results are representative also for changes obtained in other regions of the muscle.

The quantal contents of the EPSCs obtained at specific sites are indicated at the respective locations in Figure 3. Under the conditions selected for the recordings, i.e., $[\mathrm{Ca}]_{0}=6 \mathrm{mM}$ and $[\mathrm{Mg}]_{0}=30 \mathrm{mM}$, the $m$ values were between 0.1 and 1 on the nonoperated side. On the operated side, the $m$ values were larger, ranging between 0.1 and 5.5. This striking increase in the effectiveness of quantal release was always observed if the common excitor axon had been removed more than 2 days before the experiment. Searching for recording sites, the electrodes were removed along a specific muscle fiber. Positive synaptic currents were seen at more than $100 \mu \mathrm{m}$ distance from a "synaptic site" (Dudel, 1981), and the electrode then could be shifted to the maximum of steeply changing negative current (Fig. 2). Care was taken to record from all synaptic sites approached in this manner on a muscle fiber in order to eliminate bias in selection of synapses. In the operated muscle, the distance between synaptic sites along a muscle fiber is shorter then in the control side at least by a factor of two. Therefore, the $m$ values evaluated at the different sites on the operated and the control muscles should be representative of the average release conditions of the $\mathrm{L}_{1}$ axon in this region.

Figure 4 shows mean values for the quantal content obtained in seven experiments like that of Figure 3. The average quantal contents at the 100 synaptic sites in the nonoperated controls were lumped and are represented by the horizontal bar at $m=0.52$, the width of this bar corresponding to the standard error (SE) of the mean value. The abscissa in Figure 4 is days after operation, i.e., removal of the common excitor. From 2 to 23 days after the removal of common excitor, the mcan release of $\mathrm{L}_{1}$ excitor was 5 times higher than in the controls.

The effect appeared to be more or less complete within 2 days after removal of the common excitor axon. To define the onset of the reaction to removal of the common excitor more precisely, three experiments were done 1 day after the operation. In one of these experiments, no reaction was seen, while in two about half of the final level of the effect was obtained. Therefore the half-time of the development of high release by the terminals of the $L_{1}$ axon can be estimated to be between 1 and 2 days. This is a remarkably short time for a "trophic" change to manifest itself. It should be emphasized that when pronase was injected extracellularly into the space between the axons no effect was seen 10 days following the injection. In one experiment, the $\mathrm{L}_{1}$ excitor axon had been eliminated unintentionally (this axon is the smallest and most difficult to inject), and the effects of this elimination on the now remaining common excitor terminals were tested. The results were analogous to those obtained in the reverse experiment described above. Based only on this one unequivical experiment finding of the reverse direction of the effect, it seems that strengthening the release of one neuron after elimination of the other neuron may be the rule for excitatory terminals in the abdominal extensor preparation.

Changes in distribution of axon fine branches after removal of the common excitor. In addition to the increased quantal content of release, already seen as early as 1 day after elimination of the common excitor, we have also observed morphological changes which became conspicuous 4 to 5 days later. On inspection, using a dissecting microscope $(\times 20$ to 50$)$ with good illumination from a light guide, a larger number of terminal nerve branches were seen in the operated $\mathrm{L}_{1}$ muscle than in the operated controls. Incidentally, the microelectrode moving over the muscle fiber also picked up a larger number of recordings from nerve fibers in the operated muscles (e.g., see inset of Fig. 3, nonoperated). A much larger number of axonal profiles was seen in electromicrographs from operated preparations two weeks after injection (A. Dorman, I. Parnas, J. Dudel and I. Cohen, unpublished data).

To substantiate this observation, both the operated and the control preparations were stained with methylene blue for $30 \mathrm{~min}$ after each experiment. The stained preparations were observed with a Wild M5 dissecting microscope with a camera lucida attachment. Drawings 


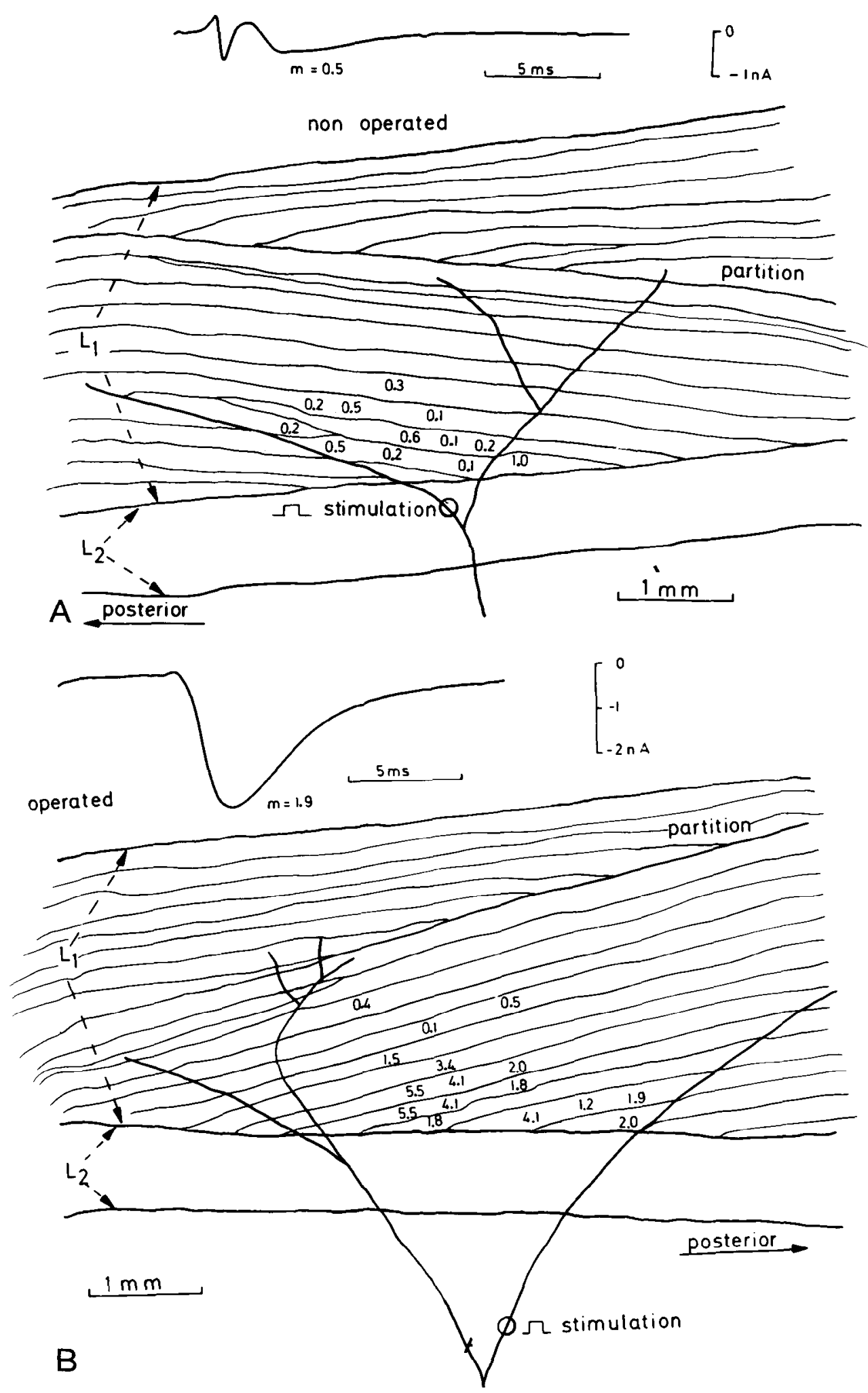

Figure 3. Increased quantal content and wider spread of excitation in $\mathrm{L}_{1}$ excitor distribution after elimination of the common excitor nerve; scheme of the $L_{1}$ muscle in the region of recording for the nonoperated $(A)$ and for the operated $(B)$ side. Only the three main nerve branches are represented. The location of the stimulating electrode is indicated near the entrance of the $\mathrm{L}_{1}$ excitor axon to the muscle. The obliquely running "partition" separates the anterior (above) and the posterior (below) portions of the $\mathrm{L}_{1}$ muscle. At the respective positions on the muscle fibers, the average quantum content $(m)$ of the recorded EPSCs is indicated. Above is a typical EPSC recorded at one of the electrode positions shown. $[\mathrm{Ca}]_{0}=6 \mathrm{mM} ;[\mathrm{Mg}]_{0}=30 \mathrm{mM}$. 


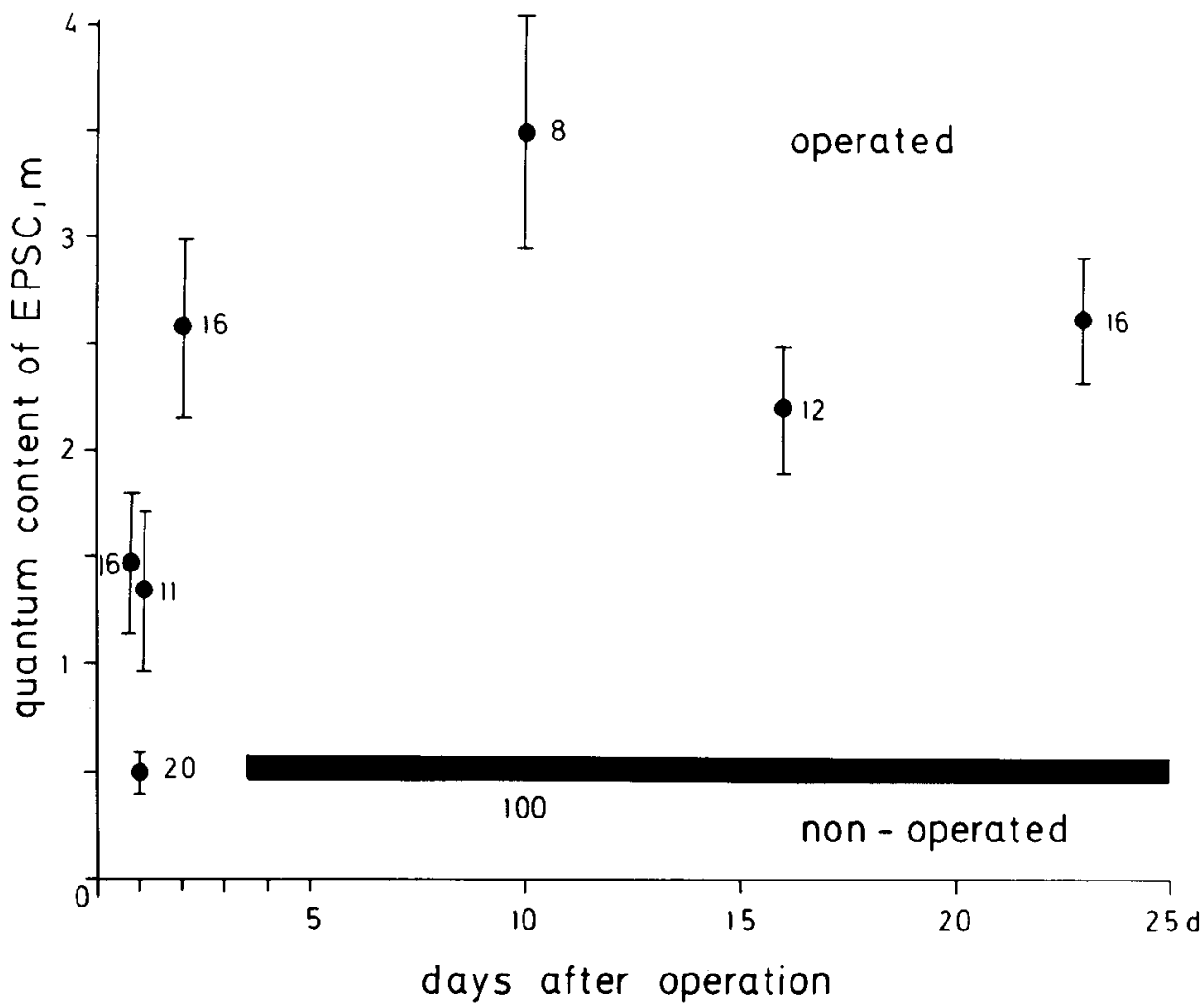

Figure 4. Time course of increase in quantal content of EPSCs; average quantal content of EPSCs ( $m$; ordinate) as a function of time after operation (abscissas), i.e., after pronase injection into the common excitor axon. the black bar indicates the average $m$ of the 100 control recordings on the nonoperated side of the $\mathrm{L}_{1}$ muscle. The width of this bar represents the standard error of the mean. The points represent average $m$ values with their standard errors of the mean measured at different times after the elimination of the common excitor. The numbers at the points indicate the number of sites at which $m$ was measured in each preparation. Each determination of $m$ is based on at least 128 single recordings. $[\mathrm{Ca}]_{0}=6 \mathrm{mM} ;[\mathrm{Mg}]_{0}=30 \mathrm{mM}$.

were made of the region of the $L_{1}$ muscle used in the experiments and of the branches of the $\mathrm{L}_{1}$ axon which had picked up methylene blue. Figure 5 shows such a drawing; few branches of the $\mathrm{L}_{1}$ axon are visible in the nonoperated preparation. This pattern of branching was constant in all nontreated muscles. However, in the preparation in which the common excitor axon had been removed 23 days before, at least 10 times as many branches were stained. In addition, some unusual branches of the $L_{1}$ excitor axon appeared on the $L_{2}$ muscle. From electrical recordings, we have no indications that the $L_{1}$ excitor axon innervated the $L_{2}$ muscle. Therefore, the branches seen on the $\mathrm{L}_{2}$ muscle must run to the $L_{1}$ muscle. A similar increase in branching was observed in all preparations more than 4 days after removal of the common excitor axon. It cannot be decided with the present evidence whether thin branches picked up staining more rapidly after the operation, whether existing branches became thicker or whether new branches sprouted. However, in electron micrographs, clear growth cone structures were seen in operated preparations at 2 weeks and never in controls (A. Dorman, I. Parnas, J. Dudel, and I. Cohen, unpublished data).

Possible role of central connections of the axons. Elimination of the common excitor could excert effects on the $\mathrm{L}_{1}$ axon terminals in a number of ways. Among them are changes occurring in the central nervous system (Rotshenker, 1979). It is also possible that the absence of the common excitor neuron stimulates the $L_{1}$ neuron to be more active or to grow. This could be accomplished either by a central or a peripheral signal or a combination of both. Another possibility is a purely peripheral functional interaction, for instance removal of a factor originating in the common excitor axon and normally depressing the $L_{1}$ excitor terminals. The simplest experiment to determine the role, if any, of central connections is to cut the bundle of five axons leading to the deep extensor muscles, after elimination of the common excitor. The cut axons will not degenerate for several months, and they remain functional, although electrically inactive. On the other hand, cutting of the $\mathrm{L}_{1}$ excitor axon would eliminate axonal continuity with its soma, which might be of importance in exerting the effect.

In three experiments, the nerve bundle to the abdominal deep extensors of segment II was cut immediately after injection of pronase into the common excitor axon. This treatment produced the unexpected result that, after 3 days, transmission in the $I_{1}$ axon was practically blocked when investigated under the same conditions as in the other experiments, i.e., $6 \mathrm{~mm}[\mathrm{Ca}]_{0}$ and $30 \mathrm{mM}$ $[\mathrm{Mg}]_{0}$. However, action potentials could be elicited in the cut $\mathrm{L}_{1}$ axon. Only when [Ca] $]_{0}$ was increased to 12 mM, approachung the normal composition of the bathing 

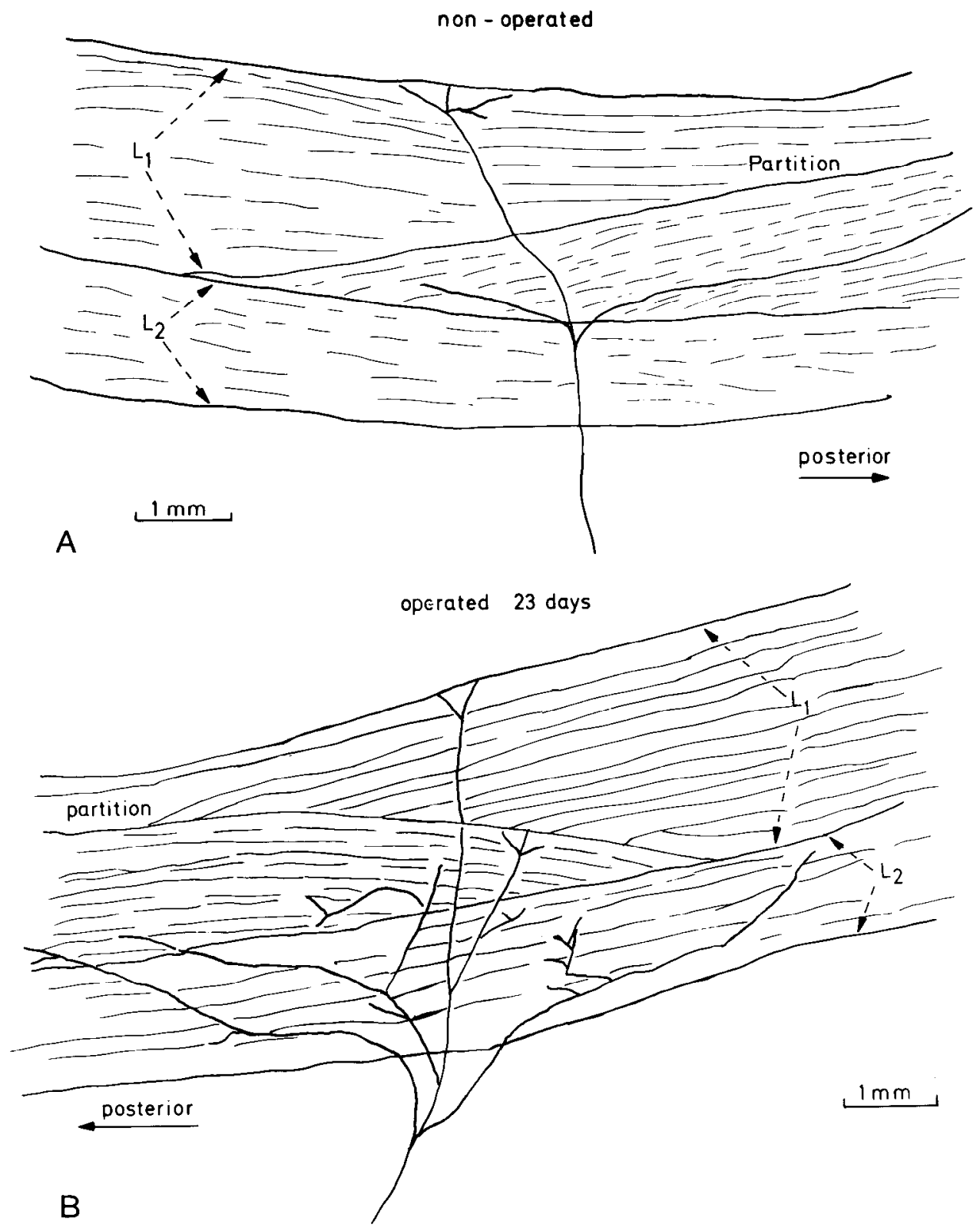

Figure 5. Increased branching of the $\mathrm{L}_{1}$ excitor nerve after elimination of the common excitor axon. These are camera lucida drawings of the region of nerve entry to the $\mathrm{L}_{1}$ and $\mathrm{L}_{2}$ muscles from the nonoperated side $(A)$ and from the side in which the common excitor axon had been eliminated 23 days before $(B)$. The preparations were stained with methylene blue for $1 / 2 \mathrm{hr}$. The drawing shows the branchings of the $L_{1}$ excitor axons which had picked up methylene blue. The profuse branches of the $L_{1}$ excitor axon on the $L_{2}$ muscle in the operated preparation do not innervate the $\mathrm{L}_{2}$ muscle but appear to proceed toward the $\mathrm{L}_{1}$ muscle.

solution, did transmission occur at the $\mathrm{L}_{1}$ excitor terminals. Therefore, in the experiment of Figure 6, such elevated $[\mathrm{Ca}]_{0}$ was applied. Consequently, the quantal content of $\mathrm{L}_{1}$ excitor EPSCs was large in the nonoperated control muscle, with an average $m=1.9$, compared to the average $m=0.52$ in the experiments of Figure 4 . In addition to the usual determination of quantal content, a qualitative measure of synaptic activity was obtained in the experiment of Figure 6. If at a specific location EPSCs with approximately normal quantal content was observed, an " $n$ " was inserted into the schematic draw- ing; if no EPSCs could be found in a specific region of a muscle fiber, a " 0 " was inserted. These additional marking allowed the characterization of larger areas of the muscle without the time-consuming determination of the quantal content in each location. If the operated muscle $(B)$ in Figure 6 is compared to the nonoperated one $(A)$, release obviously was restricted to much smaller regions than in the nonoperated control. In the operated muscle, release was usually observed only close to an axon which was either visible or from which action potentials could be recorded. In these restricted release regions, the quan- 

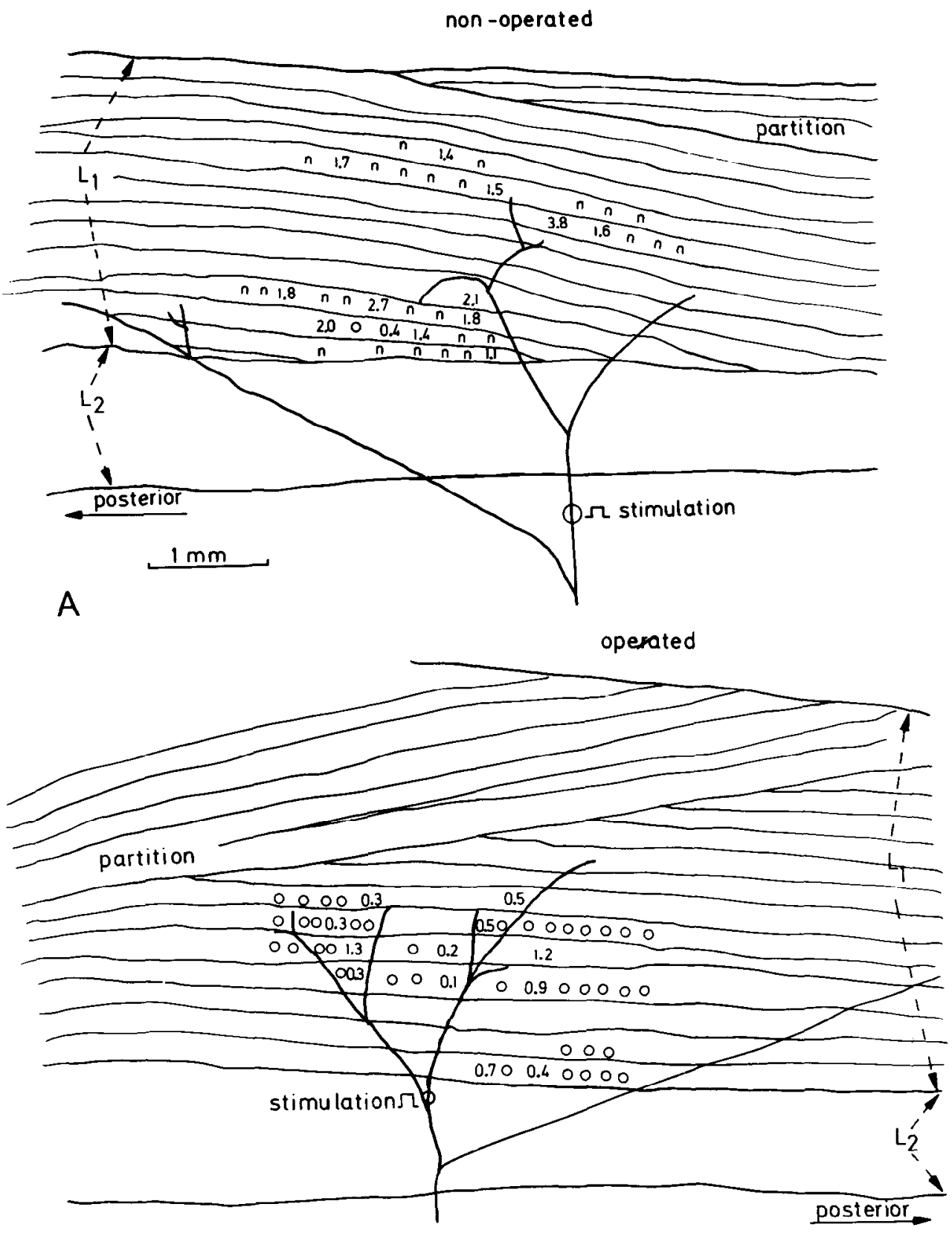

B $1 \mathrm{~mm}$

Figure 6. A decrease is seen in effective synaptic contacts and in quantal content when elimination of the common excitor is followed by decentralization of the $\mathrm{L}_{1}$ excitor axon. This scheme is of the nonoperated control muscle $(A)$ and of the contralateral operated muscle $(B)$ in which, immediately after injection of pronase into the common excitor axon, the bundle of five axons to the deep extensors was cut. The experiment was done 3 days after the operation. The numbers on the muscle fibers represent $m$ values, as in Figure 3. In addition, $n$ represents a release site with EPSCs of approximately normal quantal content, and $O$ indicates a region of the fiber in which no EPSC could be found. Other details are as in Figure 3. Note that the bathing solution contained $12 \mathrm{~mm}$ [Ca] and $10 \mathrm{mM}[\mathrm{Mg}]_{0}$. See the text.

tal content of EPSCs was lower than in the control. Therefore, it seems that the improvement of release by $\mathrm{L}_{1}$ excitor terminals due to elimination of the common excitor axon is absent when the $\mathrm{L}_{1}$ excitor is cut. However, the interpretation of this finding is complicated by the fact that in noninjected controls cutting of the $L_{1}$ excitor axon as such has a depressing effect on its ter- minals. In line with the electrophysiological results, no increase in branching of the $\mathrm{L}_{1}$ excitor axon was observed when the nerve bundle was cut after injection of the common excitor axons.

Effects of elimination of the common excitor axon on excitatory postsynaptic membrane channels. While elimination of the inhibitory axon had very strong postsyn- 
aptic effects, prolonging the EPSCs (Dudel et al., 1981; I. Parnas et al., 1982a; Ch. Franke, I. Cohen, J. Dudel, and I. Parnas, manuscript in preparation), no such effects were obvious on elimination of the common excitor. To exclude such postsynaptic actions more rigorously, single channel currents provoked by the excitatory transmitter glutamate were measured, after treatment with enzymes and concanavalin (see "Materials and Methods"). In the same region of the $L_{1}$ muscle in which EPSCs were recorded, the tip of a patch clamp electrode, which was filled with $200 \mu \mathrm{M}$ glutamate, was removed along the muscle fibers, searching for glutamate-sensitive spots. At such spots short current pulses of about -10 pA amplitude appeared (Fig. 7, inset). At these locations spontaneous synaptic miniature currents were usually also seen. Therefore, these sites are both synaptic and innervated. There was an obvious difference between the operated and the nonoperated muscles. It was easier to find a glutamate-sensitive spot in the preparations in which the common excitor axon had been eliminated (all experiments were performed more than 4 days after injection); the density of glutamate receptors or channels seemed to be higher in these preparations than in the nonoperated ones. However, the amplitudes of single channel currents were the same in operated and nonoperated preparations. The most distinctive property of a synaptic channel is its open time. Histograms of the distribution of channel open times are presented in Figure 7 for two characteristic recording sites in a nonoperated and in an operated muscle. The distributions were fitted with the sum of two exponentials, like the analogous distributions in crayfish (Ch. Franke, J. Dudel, and W. Finger, submitted for publication) and in locust muscle (Cull-Candy and Parker, 1982). The slower current component had the same time constant in the operated and in the nonoperated preparation, while the short component had a slightly smaller time constant on the operated side. This was a general finding. If there was a difference at all, the short time constant was reduced in operated muscles as compared to nonoperated ones.

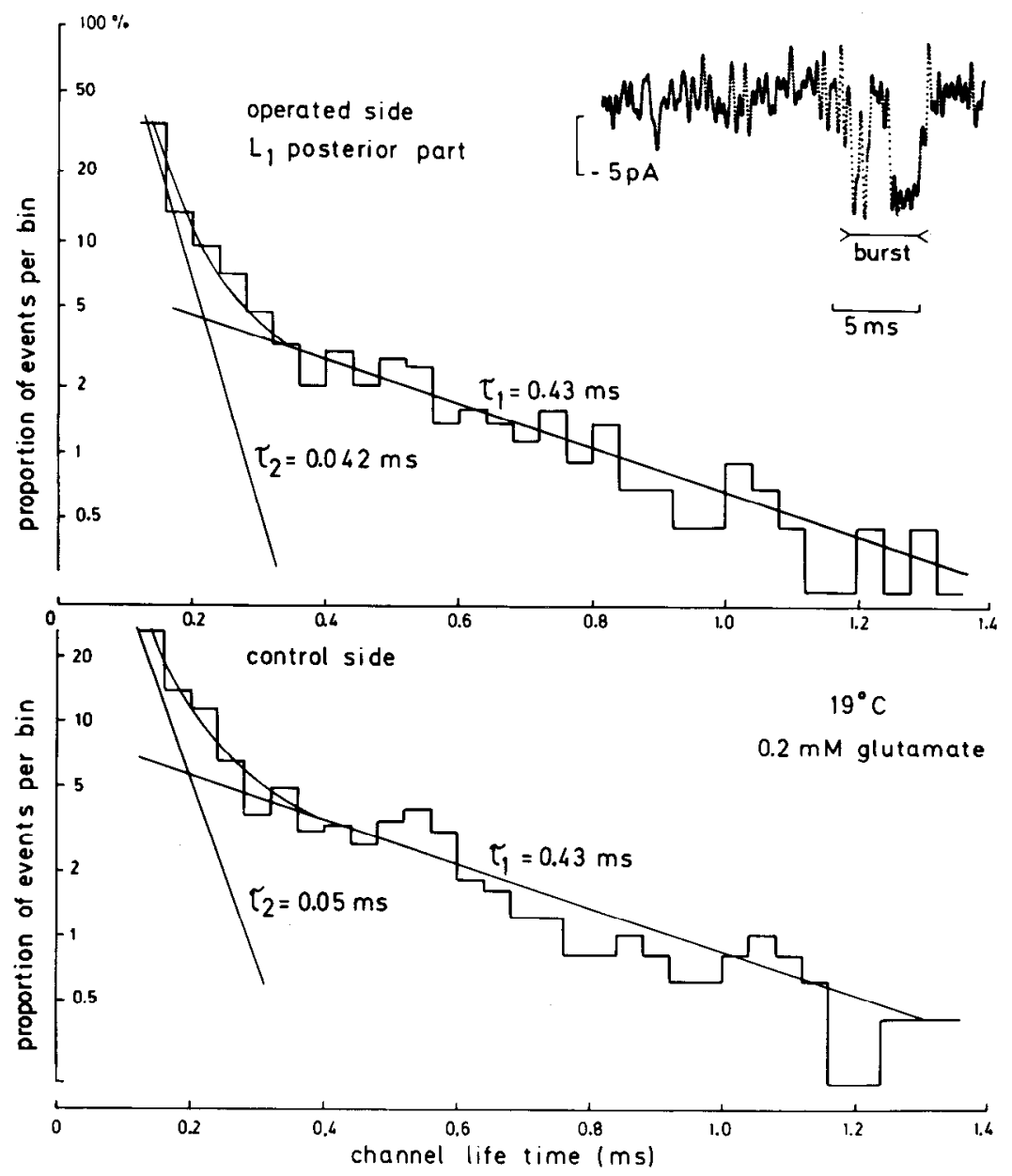

Figure 7. No unambiguous changes are seen in $\mathrm{L}_{1}$ excitatory channel open time after elimination of common excitor axon; single synaptic excitatory channel currents were elicited by $0.2 \mathrm{mM}$ glutamate in the patch clamp electrode. A sample recording is shown in the insert; seal resistance was $>100$ megohms. Distributions of channel lifetimes were measured in operated (common excitor axon eliminated) and nonoperated $\mathrm{L}_{1}$ muscles of one animal. The proportion of observed lifetimes per bin (ordinate) is plotted logarithmically; totals of 434 events were evaluated in the operated and 499 events in the nonoperated muscle. 'The distributions were fitted by the sum of two exponentials with the time constants $\tau_{1}$ and $\tau_{2}$, respectively. The average amplitude of single channel current pulses was $-11.1 \pm 1.3$ (SD) $\mathrm{pA}$. No potential was applied to the patch clamp. 
These results may be compared to the decay time of EPSCs, which were shown to agree with the slower component of the channel open time distribution in crayfish (Stettmeier et al., 1983; Ch. Franke, J. Dudel, and W. Finger, submitted for publication). In our preparations more channel openings grouped in bursts occurred than in the crayfish: in the lobster 1.8 to 1.9 openings were seen per burst, while in the crayfish the respective proportion was 1.1. Consequently, in our preparations the mean burst length was 0.8 to $0.9 \mathrm{msec}$ at $19^{\circ} \mathrm{C}$, which is a bit shorter than the decay time constant of EPSCs (Fig. 2) of about $1.3 \mathrm{msec}$ at $15^{\circ} \mathrm{C}$.

Thus, the elimination of the common excitor axon for periods of several weeks has practically no effect on the characteristics of the innervated postsynaptic excitatory membrane channels. The only obvious postsynaptic consequence of the elimination of the common excitor is the increased density of excitatory receptors and channels, which corresponds to the more dense and effective innervation by the $L_{1}$ excitor axon described above.

\section{Discussion}

Before discussing the results, we would like to stress the methodological, quantitative aspect of the present study. Usually effects of elimination of axons are registered as presence or absence of functional innervation, the criterion being contraction or intracellularly measured postsynaptic depolarization. The latter is complicated by possible changes in membrane resistance or length constant, which rarely are controlled. Extracellular recording of synaptic currents gives more detailed information; thus, the recording has spatial resolution while intracellular potentials generally are summed for the whole cell. In addition, quanta of transmitter release can be discerned better in extracellular than in intracellular records. In the present study, an increase in the effectiveness of synaptic transmission was obvious from the first experiments, and a routine was developed to allow more detailed quantitative conclusions. In this procedure, care was taken to eliminate possible bias in selection of synapses for recording. Another element in our procedure which promoted clear-cut results is the elimination of single axons by injection of pronase. This injection destroyed the axon completely, probably within hours, without damage to neighboring axons. (The pronase spreads over a distance of about $1 \mathrm{~cm}$, into the terminals within seconds after the pressure injection (I. Parnas et al., 1982a).) If axons are merely cut, degeneration proceeds within days or weeks, and usually other axons are damaged and degenerated. These side effects pose difficulties for the interpretation of results.

The main result of the present study was that removal of the strong excitatory input of the common excitor axon (Parnas and Atwood, 1966) produces changes in synaptic transmission of the weaker $L_{1}$ excitor axon. The effect does not seem to be specific for the common excitor axon. One reverse experiment indicated that elimination of the $\mathrm{L}_{1}$ excitor axon had analogous effects on transmission by the common excitor axon. This finding could be described under the heading "competition between axons innervating one cell" (Purves, 1977). It is interesting to note that terminals of the two axons are seen in electron micrographs to run close together, although profiles of single excitatory terminals, accompanied by an inhibitory terminal were also seen (A. Dorman, I. Parnas, J. Dudel, and I. Cohen, unpublished data). The results presented in this paper are another example of very specific interactions between cells of different types which could be defined with the help of the pronase injection method, demonstrated in the leech (Bowling et al., 1978; Blackshaw et al., 1982) and also in the lobster preparation by elimination of the inhibitory axon (Dudel et al., 1981; I. Parnas et al., 1982a).

The changes seen in synaptic transmission can be classified into two main categories. The first is the strengthing of release by the $L_{1}$ axon within 1 or 2 days, reaching a maximum by about 4 days. The second is sprouting of the $L_{1}$ axon to innervate the $L_{1}$ muscle more densely.

An increase in the number of quanta released per stimulus is determined presynaptically. Such an improved release could be the result of raised probability of release per single terminals as observed in synaptic facilitation (Del Castillo and Katz, 1954; Dudel and Kuffler, 1961; H. Parnas et al., 1982) or on application of 5hydroxytryptamine (Dudel, 1965; Glusman and Kravitz, 1982). Alternatively or in addition, the number of synaptic contacts of one terminal on a muscle fiber could increase. In the $L_{1}$ muscle, the axon terminals run parallel to a muscle fiber forming along their course membrane specializations with synaptic vesicles. In electron micrographs from normal preparations, we have observed sections of terminals as long as $40 \mu \mathrm{m}$, containing 10 to 12 such regions of membrane specializations, 3 to $3.5 \mu \mathrm{m}$ apart (A. Dorman, I. Parnas, J. Dudel, and I. Cohen, unpublished data). Such regions were in close conjunction to bare terminal regions. The extracellular electrode records from a membrane patch of about $20 \mu \mathrm{m}$ diameter, and a "release site" as defined by our recording technique probably corresponds to a number of synaptic contacts. This assumes that each membrane synaptic specialization as defined electron microscopically is equally active. Moreover, from the electrical measurements, we have to conclude that such regions occur about every $100 \mu \mathrm{m}$ in a normal preparation, in the region from which recordings were made. Therefore, the increased quantal content of EPSCs could be due to a higher density of synaptic contacts. Such an increase in the number of membrane specializations with clusters of synaptic vesicles was not seen in preparation even 15 days after injection, not to speak of 2 days, when 5 -fold increases were already seen. Thus, we have to conclude that the increase in synaptic release seen after a few days results from physiological changes, not accompanied with ultrastructural changes that can be discerned with our resolution of observation.

At a later stage, we find increased branching that could be traced in preparations stained with methylene blue. In electron micrographs, we find terminal profiles with clear-cut sprouting with growth cones (A. Dorman, I. Parnas, J. Dudel, and I. Cohen, unpublished data). This finding explains the higher density of synaptic innervation seen. The finding of increased density of excitatory channels corroborates this conclusion. These channels seem to lie in innervated synaptic areas; since sponta- 
neous miniature currents are recorded at low frequency, there is no evidence for extrajunctional receptors or channels and also no indication of development of channels from an immature stage with slow kinetics to an adult stage with shorter open time (Fischbach and Schuetze, 1980; Brenner and Sakmann, 1983). In a study to be reported elsewhere (J. Dudel, I. Parnas, I. Cohen, and $\mathrm{Ch}$. Franke, manuscript in preparation), we have investigated the graded depolarization release characteristics of the terminals of the $L_{1}$ excitor by the current pulse technique (Dudel, 1983). No changes in these characteristics were obvious in $L_{1}$ excitor terminals in which the common excitor axon had been removed. The EPSCs recorded in the operated muscle have a larger range of quantal content (Fig. 4), and the delays of single EPSCs seem to vary more than in normal muscles. This may indicate a larger diversity of synapses in the operated muscles, which might be expected if some of the synaptic contacts were newly formed.

What is the signal for strengthening synaptic release by the terminals of one axon on elimination of the second axon? In our case it is not the absence of the excitatory transmitter, glutamate, since all remaining excitatory terminals release glutamate. It might be argued that the reduced amount of glutamate seen by the muscle after the elimination of the common excitor axon is the trigger for strengthening of the $L_{1}$ excitor terminals, but at this stage we can neither corroborate nor rule out this possibility. Similarly, we conclude that the signal is not the absence of fiber depolarization or lack of mechanical activity, because excitatory innervation remains, although its effectiveness may be reduced. This rules out a number of possible signals, whose absence apparently serves as the trigger for increasing synaptic activity in other systems (Purves, 1977). The absence of a specific, peripheral repressing factor normally generated by the removed common excitor cannot be ruled out. Cutting of all axons to the muscle, after removing the common excitor, blocked the strengthening of release, and this block may be interpreted as excluding a peripheral signal. However, cutting of the tested $L_{1}$ excitor by itself had negative effects on the strength of transmission, and axonal continuity with the cell body may, thus, be necessary for maintaining strong synaptic contacts. But the evidence is also compatible with a purely central mediation of the strengthening of synapses on removal of the common excitor (Rotshenker, 1979). The strikingly short latent period of about 1 day until the first changes were seen is not in conflict with a central mechanism; axoplasmic transport is probably fast enough to mediate control over the short distance of 3 to $5 \mathrm{~cm}$ within a few hours. It is also possible that different signals are involved in the rapid increase in transmitter seen after 1 day and the sprouting which takes place a few days later.

The experiments in which the axons to the $L_{1}$ muscle were cut after eliminting the common excitor axon gave equivocal results with respect to the possible central control of the strengthening of release from the $L_{1}$ axon. But the observations that the field of innervation shrinks within a few days if the axon is cut, and that the rate of release decreases in the remaining terminals are interesting per se. Although the peripheral portion of the axon, without connection to the cell body, will survive for months, conduct action potentials, and maintain functional synapses (Hoy et al., 1967; Bittner and Johnson, 1974; Frank, 1974), the release at the terminals is much lower than when they are connected to the cell body. Therefore, it appears that a central factor or cofactor may control the function or the growth of terminals.

If central control mechanisms are indeed prominent, central effects could spread to the contralateral side, as observed by Rotshenker (1979) in the frog. No such effect was observed in our preparation. Numerous controls taken in nonoperated animals and the usual controls from the contralateral muscle to the operated one gave the same results. However, in preliminary experiments, cutting the nerve bundle of segment III produced increased branching in the axons of segment II on the ipsilateral side, and these sprouts even crossed contralaterally to the nonoperated side (I. Cohen and I. Parnas, unpublished data). These results support the possibility of central interaction in mediating a signal or signals for sprouting (Rotshenker, 1979).

\section{References}

Atwood, H. L. (1977) Crustacean neuromuscular systems: Past, present and future. In Identified Neurons and Behaviour of Arthropods, G. Hoyle, ed., pp. 9-29, Plenum Publishing Corp., New York.

Atwood, H. L., and I. Kwan (1976) Synaptic development in the crayfish opener muscle. J. Neurobiol. 7: 289-312.

Brenner, H. R., and B. Sakmann (1983) Neurotrophic control of channel properties at neuromuscular synapses of rat muscle. J. Physiol. (Lond.) 337: 159-171.

Blackshaw, S. F., J. G. Nicholls, and I. Parnas (1982) Fxpanded receptive fields of cutaneous mechanoreceptor cells after single neuron deletion in leech central nervous system. J. Physiol. (Lond.) 326: 261-268.

Bittner, G. D., and A. L. Johnson (1974) Degeneration and regeneration in crustacean peripheral nerves. J. Comp. Physiol. 89: 1-21.

Bowling, D., J. G. Nicholls, and I. Parnas (1978) Destruction of a single cell in the central nervous system of the leech as a means of analysing its connexions and functional role. $J$. Physiol. (Lond.) 282: 169-180.

Brown, M. C., J. K. S. Jansen, and D. E. Van Essen (1976) Polyneuronal innervation of skeletal muscle in new-born rats and its elimination during maturation. J. Physiol. (Lond.) 261: $387-422$.

Cull-Candy, S. G., and I. Parker (1982) Rapid kinetics of single glutamate-receptor channels. Nature 295: 410-412.

Del Castillo, J., and B. Katz (1954) Statistical factors involved in neuromuscular facilitation and depression. J. Physiol. (Lond.) 124: 574-585.

Dudel, J. (1965) Facilitatory effects of 5-hydroxy-tryptamine on the crayfish neuromuscular junction. Naunyn-Schmiedebergs Arch. Exp. Pathol. Pharmakol. 249: 515-528.

Dudel, J. (1979) The voltage dependence of the decay of the excitatory postsynaptic current and the effect of concanavalin $\mathrm{A}$ at the crayfish neuromuscular junction. J. Physiol. (Paris) 75: 601-604.

Dudel, J. (1981) The effect of reduced calcium on quantal unit current and release at the crayfish neuromuscular junction. Pflugers Arch. 391: 35-40.

Dudel, J. (1983) Graded or all-or-nothing release of transmitter quanta by local depolarization of nerve terminals on crayfish muscle? Pflugers Arch. 398: 155-164. 
Dudel, J., and S. W. Kuffler (1961) Mechanism of facilitation at the crayfish neuromuscular junction. J. Physiol. (Lond.) 155: 530-542.

Dudel, J., Y. Grossman, and I. Parnas (1981) Synaptic transmission in crustacean muscle: Effects of elimination of the inhibitor fiber on excitatory transmission. In Amino Acid Neurotransmitters, F. V. DeFeudis and P. Mandel, eds., pp. 359-364, Raven Press, New York.

Dudel, J., I. Parnas, and H. Parnas (1982) Neurotransmitter release and its facilitation in crayfish. III. Amplitude of facilitation and inhibition of entry of calcium into the terminal by magnesium. Pflugers Arch. 393: 237-242.

Fischbach, G. D., and S. M. Schuetze (1980) A post-natal decrease in acetylcholine channel open time at rat end-plates. J. Physiol. (Lond.) 303: 125-137.

Frank, E. (1974) The sensitivity to glutamate of denervated muscles of the crayfish. J. Physiol. (Lond.) 242: 371-382.

Glusman, S., and E. A. Kravitz (1982) The action of serotonin on excitatory nerve terminals in lobster nerve-muscle preparations. J. Physiol. (Lond.) 325: 223-241.

Haimann, C., A. Mallart, J. Tomas I Ferre, and N. F. ZilberGachelin (1981) Patterns of motor innervation in the pectoral muscle of adult Xenopus laevis: Evidence for possible synaptic remodelling. J. Physiol. (Lond.) 310: 241-256.

Hoy, R. R. (1969) Degeneration and regeneration in abdominal flexor motoneurons in the crayfish. J. Exp. Zool. 172: 219232.

Hoy, R. R., G. D. Bittner, and D. Kennedy (1967) Regeneration in crustacean motoneurons: Evidence for axonal fusion. Science 156: 251-252.

Hume, R. J., and D. Purves (1983) Apportionment of the terminals from single preganglionic axons to target neurons in the rabbit ciliary ganglion. J. Physiol. (Lond.) 338: 259275.

Jenkinson, D. H. (1957) The nature of antagonism between calcium and magnesium ions at the neuromuscular junction. J. Physiol. (Lond.) 138: 434-444.

Johnson, E. W., and A. Wernig (1971) The binomial nature of transmitter release at the crayfish neuromuscular function. J. Physiol. (Lond.) 218: 757-767.

Katz, B., and R. Miledi (1965) The effects of calcium on acetylcholine release from motor nerve terminals. Proc. $\mathrm{R}$. Soc. Lond. Biol. 161: 496-503.

Mathers, D. A. (1981) The influence of concanavalin A on glutamate-induced current fluctuations in locust muscle fibres. J. Physiol. (Lond.) 312: 1-8.

Neher, E., B. Sakmann, and J. H. Steinbach (1978) The extracellular patch clamp: A method for resolving currents through individual open channels in biological membranes. Pflugers Arch. 375: 219-228.

Parnas, H., J. Dudel, and I. Parnas (1982) Neurotransmitter release and its facilitation in crayfish. I. Saturation kinetics of release and of entry and removal of calcium. Pflugers Arch. 393: 1-14.

Parnas, I., and H. L. Atwood (1966) Phasic and tonic neuromuscular systems in the abdominal extensor muscles of the crayfish and rock lobster. Comp. Biochem. Physiol. 18: 701723.

Parnas, I., and D. Bowling (1977) Killing of single neurons by intracellular injection of proteolytic enzymes. Nature 270 : 626-628.

Parnas, I., J. Dudel, and Y. Grossman (1982a) Chronic removal of inhibitory axon alters excitatory transmission in a crustacean muscle fiber. J. Neurophysiol. 47: 1-10.

Parnas, I., H. Parnas, and J. Dudel (1982b) Neurotransmitter release and its facilitation in crayfish. II. Duration of facilitation and removal processes of calcium from the terminal. Pflugers Arch. 393: 232-236.

Purves, D. (1977) The formation and maintenance of synaptic connections. In Function and Formation of Neural Systems., G. S. Stent, ed., pp. 21-41, Dahlem Konferenzen, Berlin.

Purves, D., and J. W. Lichtman (1978) Formation and maintenance of synaptic connections in autonomic ganglia. Physiol. Rev. 58: 821-862.

Purves, D., and J. W. Lichtman (1980) Elimination of synapses in the developing nervous system. Science 210: 153-157.

Rotshenker, S. (1979) Synapse formation in intact innervated cutaneous pectoris muscles of the frog following denervation of the opposite muscle. J. Physiol. (Lond.) 292: 535-547.

Stettmeier, H., W. Finger, and J. Dudel (1983) Glutamate activated postsynaptic channels in crayfish muscle investigated by noise analysis. Pflugers Arch. 397: 13-19. 